\title{
Cancer institute to tighten control of trials
}

Washington. The US National Cancer Institute (NCI) plans to strengthen its monitoring of clinical trials following the recent public outcry over the discovery of fraudulent data in a trial evaluating the treatment of women with breast cancer.

Samuel Broder, director of the NCI, last week told the House of Representatives' subcommittee on oversight and investigation, chaired by John Dingell (Democrat, Michigan), that he accepted responsibility for the fact that the NCI had not been quicker in sorting out problems with the trial.

But he told Dingell that a reanalysis by the NCI of the original clinical trials indicated that the fraudulent data did not invalidate the conclusion of the trial. This showed that lumpectomy followed by radiation therapy is a preferable treatment for most women with early cancer to the more disfiguring and physically disabling mastectomy.

The lumpectomy trial is one of several exploring treatment for breast cancer, each involving many hospitals, that are being coordinated by the National Surgical Adjuvant Breast and Bowel Project (NSABP), set up 25 years ago at the University of Pittsburgh under the leadership of Bernard Fisher.

Several other large trials have reached the same conclusions as the NSABP. But women's health advocates told Dingell's committee that the previous lack of openness about the fraudulent data, which were submitted to the NSABP by Roger Poisson of St Luc's Hospital in Montreal, meant that many women are now worried about their decision to choose a lumpectomy.

Last year, the Department of Health and Human Services' Office of Research Integrity (ORI) concluded that Poisson altered the records of 15 per cent of his patients over 13 years to make them eligible for inclusion in the trial. Concern about the NSABP's failure to spot this earlier was heightened at the end of last month when NCI officials found a discrepancy in data submitted for another of the project's clinical trials. These came from St Mary's Hospital, also in Montreal, and arose from a trial of the effectiveness of the drug tamoxifen in preventing breast cancer in women known to be at a high risk of developing the disease.

The ORI is now investigating the tamoxifen data, and the NCI has asked the University of Pittsburgh to replace Bernard Fisher - who some believe is being made a scapegoat for the whole affair — as the NSABP's principal investigator.

Broder stressed that Fisher was being replaced for administrative failures, not scientific misconduct. The $\mathrm{NCI}$ is annoyed that Fisher took nine months to inform them of problems with data in the lumpectomy trial, and failed to notify them when his staff found discrepancies in the tamoxifen trial.

It is also upset that he did not promptly submit for publication a reanalysis of the lumpectomy trial data excluding Poisson's contribution, and that the NSABP last year stopped checking samples of the data points it received against the originals held by hospitals, a standard technique used to monitor the quality of the data that hospitals send in to groups controlling large clinical trials

As a result, the NCI told the NSABP at the end of March to suspend recruitment to all of its trials. NCI staff are now reviewing

\section{BASF opens biotech laboratory in the US}

Munich. The chemical company BASF last week formally opened its first major pharmaceutical research centre outside Germany, in Worcester, Massachusetts. The centre will carry out research in cancer and diseases of the immune system, and manufacture some biopharmaceutical products.

The decision to build the US\$100-million BASF Bioresearch Corporation (known as $B B C$ ) in the United States was partly a response to the difficulties that chemical companies have experienced in manufacturing bioengineered products in Germany. In 1988, BASF's plans to build a production plant for recombinant $\mathrm{TNF} \alpha$, a potential antitumour agent, were blocked because of the country's strict gene laws. The company decided to transfer biotechnology research across the Atlantic, but, not everything has gone according to plan. Falling profits in BASF's pharmaceutical division have slowed down the development of the centre. It opened with 160 staff and will not reach its full capacity of 450 for several years.

Furthermore, the centre's original aim of developing and producing mostly proteinbased therapeutics has been modified as a result of the disappointing clinical performance of many such compounds. As a result, the centre's biotechnology production facilities, currently being used only to manufacture BASF's new anticoagulant PEGhirudin, are underutilized.

$\mathrm{BBC}$ is seeking partners to rent out excess capacity to other companies. But its president, Robert Kamen, says he is not disappointed by the scaled down plans, which he describes as "realistic". Alison Abbott all of the original data at the 400 participating sites of the lumpectomy trial.

Fisher, who is in his mid-seventies, was too ill to attend last week's hearing. But he released a statement saying that, if there had been any evidence that the conclusions of the NSABP studies were affected by the St Luc's data alterations, he would have reported it immediately. "In retrospect, this proved to be a mistake," said Fisher. "The lack of publication raised unnecessary fears among breast cancer patients."

Fisher has a number of supporters. Richard Peto, for example, professor of medical statistics and epidemiology at the University of Oxford, has sent a letter backing him to the New England Journal of Medicine. Peto says he is particularly concerned that more aggressive auditing of data will add heavy costs to large-scale randomized clinical trials, even though these are essential for uncovering effects that are small yet significant for treatment.

But in an attempt to allay public criticism arising from the Poisson case, Broder told the congressional subcommittee last week that the NCI would make more unannounced spot checks on hospitals participating in large trials. In particular, the institute will focus on the new tamoxifen trial, which is already highly contentious in both the United States and Britain. Tamoxifen is known to reduce the occurrence of new cancers in women already treated for breast cancer, and researchers hope that it may prevent cancer in women at high risk of contracting breast cancer. But the drug has resulted in death from cancer of the uterine lining in some patients.

The NSABP was informed of the first such death in 1991, but did not inform the NCI until August 1993. This information is only now being included in informed consent forms that women sign before a trial.

Under close questioning by Dingell, Broder accepted that the NSABP could have passed this information to the NCI in early 1992. He promised that the institute will improve the flow of data about drug toxicity between itself and bodies such as the NSABP that manage and analyse large clinical trials.

Researchers still face the question of whether the new data mean that tamoxifen should be withheld from healthy women at high risk of breast cancer, even though the benefit still outweighs the risk for women who have already had breast cancer.

Last week, the Food and Drug Administration required that tamoxifen be relabelled with a warning that it poses a higher risk of cancer of the uterus than previously thought. Ed Sondik, acting deputy director of the NCI, says the institute will evaluate the information now available to decide whether the criteria for participating in the trial should be altered. Helen Gavaghan 